\title{
SYNCHRONOUS ENDOMETRIAL AND OVARIAN ADENOCARCINOMAS
}

\author{
Hemalatha. A. L, Sumana Sindhuram, Umarani. M. K, Suma. J. K, Deepthi. B. R.
}

1. Professor \& HOD, Department of Pathology, Mysore Medical College \& Research Institute.

2. Assistant Professor, Department of Pathology, Mysore Medical College \& Research Institute.

3. Assistant Professor, Department of Pathology, Mysore Medical College \& Research Institute.

4. PG Student, Department of Pathology, Mysore Medical College \& Research Institute.

5. PG Student, Department of Pathology, Mysore Medical College \& Research Institute.

\section{CORRESPONDING AUTHOR}

Dr. Hemalatha. A. L. $156,12^{\text {th }}$ cross, $2^{\text {nd }}$ main, Jayanagar, Mysore-570014, Karnataka.

E-mail: hlingappa@rediffmail.com

Ph: 0821-2567505.

ABSTRACT: Rare instances of endometrial \& ovarian cancers presenting at the same time (synchronous) have been reported in literature with their incidence rate ranging from $2-8.5 \%$. They may or may not share the same histologic features. When they share identical histology, it is difficult to categorize them as two independent primaries or as one primary with metastasis in the other.

Despite the difficulty, prognostication depends on this differentiation since two independent primary tumors with identical histology carry a better prognosis than the other category.

Molecular genetics may be the sole answer when such a difficulty is encountered. We present one such quizzical case of synchronous endometrial \& ovarian adenocarcinomas with similar histology occurring in a 51 year old female.

KEYWORDS: Adenocarcinoma, Endometrial, Ovarian, Synchronous

INTRODUCTION: Synchronous endometrial and ovarian cancers are infrequently found with incidence rate of $2-8.5 \% \cdot{ }^{[1,2,3]}$ They may have similar or different histologic appearances. Rare cases of papillary, serous, clear cell carcinomas and carcinosarcoma of endometrium with ovarian primary have been reported.[1,4]

Patients with tumors of identical histology at both sites should be categorised as either two individual primaries or as primary at one site with metastasis at the other site. The diagnosis of synchronous ovarian and endometrial cancer is less in doubt when tumors have dissimilar histology, as majority of them are cases of metastasis to ovary from endometrial primary.[1]

CASE REPORT: A 51 year old female patient, $\mathrm{P}_{3} \mathrm{~L}_{3}$, presented with complaints of lower abdominal mass, menorrhagia, irregular inter-menstrual bleeding and lower abdominal pain. The abdominal mass was of 20 weeks' size and was also felt per vaginum.

Ultrasound examination of pelvis showed enlarged uterus with bilateral ovarian masses. Following a clinical diagnosis of fibroid uterus associated with bilateral ovarian masses, the patient underwent total abdominal hysterectomy with bilateral salphingo-oophorectomy \& the specimen was subjected to histopathological examination. 
GROSS EXAMINATION FINDINGS: The uterus was bulky \& cervix hypertrophied. On sectioning, a tiny intramural fibroid was found. Endometrium was thickened, shaggy, irregular and friable. The right ovarian mass with overlying stretched fallopian tube measured $13 \times 11 \times 6 \mathrm{~cm}$ and the left one measured $22 \times 16 \times 14 \mathrm{~cm}$. Outer surface of both the masses were smooth. On sectioning, both exuded dark brown serous fluid and showed multiple, irregular grey brown solid areas and a few cystic areas (Fig 1).

Extensive sampling was done from uterus, cervix, bilateral ovarian masses \& fallopian tubes.

MICROSCOPIC FINDINGS: Endometrium showed increased gland to stromal ratio and back to back arrangement of glands with complex branching, villoglandular \& glandular patterns (Figure 2). Some non-villous papillae were also seen. A solid pattern was seen in about $25 \%$ of the total area. The lining epithelium showed stratification, loss of nuclear polarity, atypia, high nuclear-cytoplasmic ratio, nuclear hyperchromasia and abnormal mitotic figures. Foci of squamous metaplasia, stromal invasion and desmoplasia were seen. Myometrium was free from invasion. Endocervix showed tumor infiltration. The solid areas in the bilateral ovarian masses showed the same features as the endometrial tumor (Figure 3). The cystic areas showed features of serous cystadenoma. Fallopian tubes were free from invasion.

Based on these findings, a final diagnosis of synchronous endometrial and ovarian adenocarcinoma of endometrioid type with squamous metaplasia \& tumor extension into endocervix associated with bilateral ovarian serous cystadenoma was arrived at.

DISCUSSION: Synchronous endometrial and ovarian primary cancers are infrequently found in women undergoing surgery and the reported incidence rates range from 2 to $8.5 \%$. Patients with tumor in both the endometrium and ovary can be classified into 3 groups namely, endometrial cancer with metastasis to ovary, two independent primary tumors \& ovarian cancer with metastasis to the endometrium, which is the least common. ${ }^{[5]}$

Majority of the synchronous tumors are adenocarcinomas. Rare cases of uterine carcinosarcomas and leiomyocarcinomas associated with ovarian carcinomas have been reported. ${ }^{[1]}$ The two tumors may have similar appearances, which are in most instances, endometrioid type of adenocarcinomas as in the present case. They may even be of different histologic types, as in an endometrioid type of endometrial cancer associated with serous ovarian cancer.[1,5]

Most neoplasms with endometrioid appearance at both sites are probably independent neoplasms. These tumors have a better overall prognosis as compared to those with nonendometrioid appearance and also those with a single primary at one site \& metastasis at the other.

Non-endometrioid type of synchronous tumors, especially papillary serous carcinomas are high grade tumours with early peritoneal and distant metastasis.[4]

The inherent problem however, in patients with tumor of identical histology at both sites as in the present case, is to categorise them as individual primary tumors or as one primary with metastasis to the other site. Because, the former has better prognosis than the latter. In contrast, the diagnosis of a synchronous ovarian and endometrial cancer is less in doubt when the tumors have dissimilar histology, as majority of them are cases of metastasis to ovary from a primary endometrial tumor.,[1,5] 
Various criteria have been proposed to distinguish between ovarian metastasis from primary endometrial carcinoma and two independent primaries. ${ }^{[6,8,9]}$ Nonetheless, the ovary is a known site of metastatic spread from uterus. ${ }^{[10]}$ So, many of the ovarian tumors may simply represent endometrial metastasis.

Factors favoring two independent primary tumors are, histologic dissimilarity of the tumor, only superficial myometrial or no invasion, no vascular space invasion of endometrial tumor, absence of other evidences of spread of endometrial tumor, ovarian tumor unilateral (80-90\% of cases), surface implants or predominant hilar location in ovary, presence of ovarian endometriosis, different ploidy of DNA indices, dissimilar molecular genetics or karyotypic abnormalities in the tumors. ${ }^{[5,8]}$

Factors favoring endometrial primary and ovarian secondary are, histologic similarity of the tumors, large endometrial tumor- small ovarian tumor/s, deep myometrial ovarian and vascular space invasion, spread elsewhere of endometrial carcinoma, bilateral and/or multiple ovarian tumors, hilar location, surface implants or combination in ovary, absence of ovarian endometriosis, aneuploidy with similar DNA indices or diploidy of both tumors, similar molecular genetics or karyotypic abnormalities.[5,8]

Since the present case had considerable overlap of features from both these categories, molecular genetics seems to be the only option to decide about which category they belong to. The patient was lost for follow-up since she was referred to an advanced medical centre for management.

Pamela T. Saliman, Brian M. Slomavitz, Russell R. Broadders et al found that women with synchronous primary cancers of the endometrium and ovary had distinct clinical features such as, young age at presentation, obesity, premenopausal status and multiparity. ${ }^{[5]}$ All these features except the age factor were concurrent with the present case.

CONCLUSION: Among the synchronous uterine \& ovarian neoplasms, those with endometrioid histology at both sites have an excellent overall prognosis as compared to those with nonendometrioid histology. Hence, there is an absolute need to distinguish between the two groups for the purpose of prognostication and to decide whether the two tumors are independent primaries, or whether one is a primary \& the other a secondary, in which case, which one of them is the primary?

\section{REFERENCES:}

1. Iris M. Castro, Philip P.Connel, Steven Waggoner, Jacob Rotmensch and Arno J. Mundt. Synchronous Ovarian and Endometrial Malignancies. Ani J Clin Oncol (CCT) 2000; 521525.

2. Anneger JF, Malleasian GD. Patterns of other neoplasms in patients with endometrial carcinoma. Cancer 1981; 48: 856-9.

3. Kottmeier HL. The diagnosis and treatment of ovarian malignancies. Arch Pathol 1965; 37:51-64.

4. Juan Rosai. Rosai and Ackerman's surgical pathology. 9 th ed vol 2: Mosley An Imprint by Elsevier; 2004.

5. Pamela T. Soliman, Brian M. Slomovitz, Russel R. Broadders, Charlotte C Sun, Jonathan C Oh, Patricia J. Elifel, David M. Gershenson and Karan H. hu. Synchronous primary cancers 
of the endometrium and ovary: a single institution review of 84 cases. Gynecology Oncology 2004; 94: 456-462.

6. Ulbright TM, Roth LM. Metastatic and independent cancers of the endometrium and ovary: a clinicopathologic study of 34 cases. Hum Pathol 1985; 16: 28-34.

7. Jambhekar NA, Sampat MB. Simultaneous endometrioid carcinoma of the uterine corpus and ovary: a clinicopathologic study of 15 cases. J Surg Oncol 1988; 37: 2023-8.

8. Scully RE, Young RH, Clement PB. Tumors of the ovary, maldeveloped glands, fallopian tube, and broad ligament, Atlas of tumor pathology. Bethesda, MD: Armed Forces Institute of Pathology; 1998.

9. Shen B-C, Lin $\mathrm{H}-\mathrm{H}$, Chen $\mathrm{C}-\mathrm{K}$, et al. Synchronous primary carcinomas of the endometrium and ovary. Int J Gynecol Obstet 1995; 51: 141-6.

10. Warren S, Gates O. Multiple primary malignant tumors. Am J Cancer 1932; 16: 1358-414.

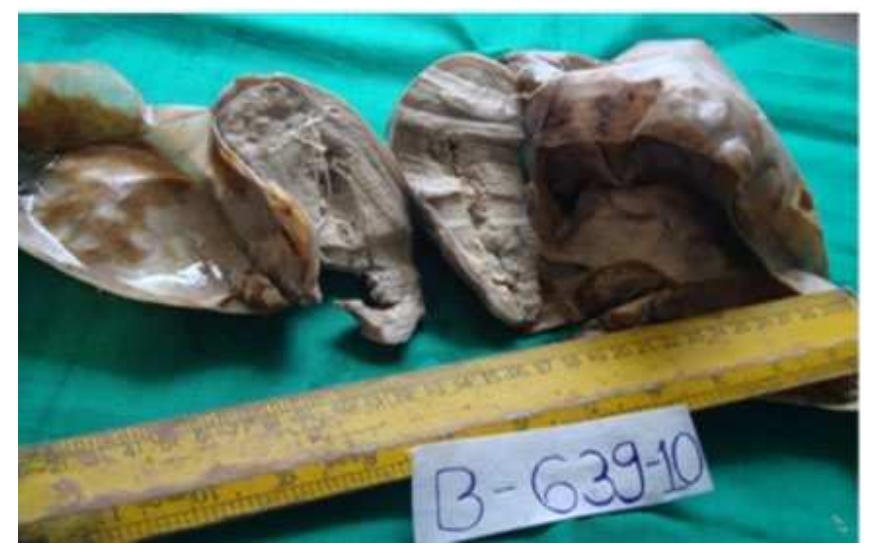

\section{Irregular endometrial growth with bilateral partly solid and cystic ovarian tumor}

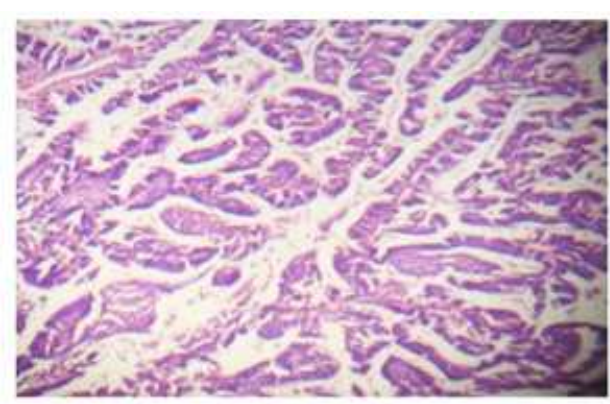

Malignant endometrial glands with back to back arrangement,complex branching, villoglandular and glandular patterns. $x$ 40X(H\&E)

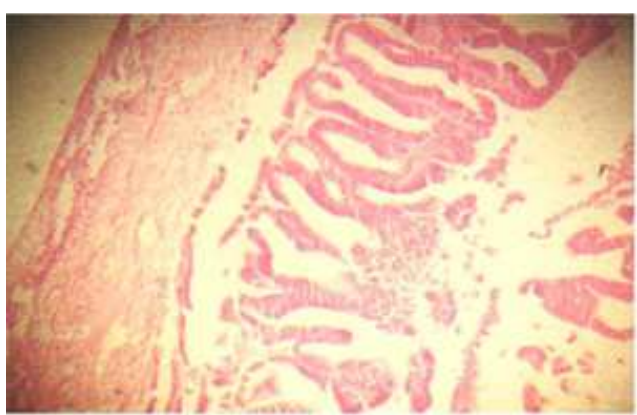

Ovarian endometriod adenocarcinoma histologically similar to endometrial adenocarcinoma.x10X(H\&E) 